\title{
Simulação do modelo constitutivo integral KBKZ-PSM usando diferenças finitas em malhas hierárquicas
}

\author{
Juliana Bertoco ${ }^{1}$ \\ Departamento de Matemática, UFSCar, São Carlos, SP. \\ Rosalía Taboada Leiva ${ }^{2}$ \\ Departamento de Matemática Aplicada e Estatística, ICMC, USP, São Carlos, SP. \\ Antonio Castelo Filho ${ }^{3}$ \\ Departamento de Matemática Aplicada e Estatística, ICMC, USP, São Carlos, SP.
}

Resumo. Esse trabalho apresenta um método numérico para resolver as equações governantes para escoamentos viscoelásticos modelados pela equação constitutiva integral KBKZPSM em malhas hierárquicas. A metodologia proposta é verificada num escoamento entre placas paralelas. Os resultados numéricos nas malhas avaliadas mostraram-se em concordância com a solução semi-analítica.

Palavras-chave. Modelo constitutivo integral KBKZ, método dos campos de deformação, diferenças finitas, malhas hierárquicas.

\section{Introdução}

Através dos anos tem-se desenvolvido vários softwares para a resolução de problemas que envolvem escoamentos de fluidos viscoelásticos complexos. Devido a falta de generalidade, alguns problemas podem surgir quando tenta-se resolver problemas com características específicas. Na maioria dos trabalhos que desenvolvem métodos numéricos para simulação de escoamentos viscoelásticos, as equações constitutivas são aproximadas por equações diferenciais, como por exemplo, os modelos UCM, Oldroyd-B, Phan-ThienTanner, FENE-CR (FENE-P), eXtended Pom-Pom, entre outros. No entanto, os avanços em recursos computacionais têm motivado pesquisadores a considerar modelos reológicos mais sofisticados que empregam equações integrais ao invés de equações diferenciais. Neste sentido, os modelos integrais, permitem uma melhor aproximação do comportamento de fluidos viscoelásticos, entretanto, exigem um maior esforço computacional; pois em cada momento da simulação, tem-se que armazenar e acessar o histórico de toda a deformação do fluído (desde que ele começou a ser deformado).

\footnotetext{
${ }^{1}$ julianabertoco@dm.ufscar.br

${ }^{2}$ rosalia.taboada@usp.br

${ }^{3}$ castelo@icmc.usp.br
} 
Entre os modelos integrais investigados, a equação constitutiva KBKZ-PSM têm sido considerada por muitos pesquisadores que estudam métodos numéricos para esta classe de fluidos. Uma detalhada discussão sobre a importância do modelo constitutivo integral KBKZ-PSM e o desenvolvimento das técnicas numéricas para aproximar modelos integrais podem ser encontradas nos trabalhos de Tanner [10] e Mitsoulis [7]. A grande maioria dos problemas utilizando o modelo integral KBKZ-PSM envolvem escoamentos confinados, como por exemplo fluxos em canais [1,5] e fluxos em contrações abruptas [6]. Por outro lado, solução numérica de equações diferenciais parciais em grades gerais tem sido indagada por muitos pesquisadores nas últimas décadas. Muitos esquemas tentam combinar eficiência e simplicidade com a flexibilidade de redes de malhas não estruturadas. Uma grande vantagem é a capacidade de refinar localmente a malha, melhorando a precisão em regiões específicas sem aumentar drasticamente o número de incógnitas. Entre todas as formas possíveis de discretizar o domínio espacial (malhas simplificadas, malhas curvilíneas, entre outras), as malhas hierárquicas baseadas em árvores cartesianas são uma escolha comum. Elas permitem o desenvolvimento de métodos de diferenças finitas, sem o incômodo de mapear e transformar elementos distorcidos ou lidar com estênceis gerais e complicados, como acontece em grades não-cartesianas. Como os fluxos são geralmente computados em facetas alinhadas com o eixo cartesiano, esquemas numéricos são geralmente mais simples de derivar. Ainda assim, essas facetas são geralmente compartilhadas por diferentes números de elementos em cada lado, o que é a principal dificuldade para métodos numéricos. Diferentes técnicas para lidar com este problema foram desenvolvidas na literatura, a maioria delas restritas a malhas quadtree (em 2D) ou octree (em 3D), que são casos especiais de grades hierárquicas representadas por estruturas de dados quadtree/octree. Apesar dessa restrição, essas estruturas de dados baseadas em árvore são gerais o suficiente e ainda uma escolha adequada para grades adaptativas e fronteiras móveis [4]. Assim, neste trabalho, pretende-se implementar o modelo KBKZ-PSM através de um método de diferencias finitas em malhas hierárquicas que empregam interpolações usando mínimos quadrados móveis (MQM).

\section{Equações Governantes}

Escoamentos incompressíveis de fluidos viscoelásticos são descritos pelas equações de conservação da massa e de conservação de quantidade de movimento dadas, respectivamente, por

$$
\begin{gathered}
\nabla \cdot \mathbf{u}=0 \\
\frac{\partial \boldsymbol{u}}{\partial t}+\nabla \cdot(\boldsymbol{u u})=-\nabla p+\frac{1}{R e} \nabla^{2} \boldsymbol{u}+\nabla \cdot \mathbf{\Phi}+\frac{1}{F r^{2}} \boldsymbol{g}
\end{gathered}
$$

nas quais $\boldsymbol{u}$ é o vetor velocidade, $p$ é a pressão, $\boldsymbol{g}$ é o campo gravitacional. Nestas equações adimensionais, $R e=\frac{\rho L U}{\eta_{0}}$ onde $\rho$ é a densidade e $\eta_{0}$ é a viscosidade total, $L$ e $U$ são, respectivamente, as escalas de comprimento e de velocidade características, e $F r=\frac{U}{\sqrt{L g}}$. Na equação (2), $\boldsymbol{\Phi}$ é um tensor não Newtoniano que é derivado do tensor de tensões extra $\boldsymbol{\tau}$ por $\boldsymbol{\Phi}=\boldsymbol{\tau}-\frac{\left(1-\beta_{s}\right)}{R e} \dot{\gamma}$ onde $\dot{\gamma}=\nabla \mathbf{v}+(\nabla \mathbf{v})^{T}$ e $\beta_{s}$ é a contribuição de solvente. 
Neste trabalho, tensor de tensão extra $\tau$ é descrito pelo modelo reológico integral $\mathrm{K}-\mathrm{BKZ}[2]$ que pode ser expresso como

$$
\boldsymbol{\tau}=\int_{-\infty}^{t} M\left(t-t^{\prime}\right) H\left(I_{1}, I_{2}\right) \boldsymbol{B}_{t^{\prime}}(t) d t^{\prime}
$$

onde $\boldsymbol{B}$ é o tensor de Finger;

$$
H\left(I_{1}, I_{2}\right)=\frac{\alpha}{\alpha+\beta I_{1}+(1-\beta) I_{2}},
$$

é a função de decaimento de Papanastasiou-Scriven-Macosko [8], $\alpha$ e $\beta$ são parâmetros do fluido e $I_{1}$ e $I_{2}$ denotam o primeiro e segundo invariantes definidos por $I_{1}=\operatorname{tr}(\boldsymbol{B})$, e $I_{2}=$ $\frac{1}{2} \operatorname{tr}\left[I_{1}^{2}-\operatorname{tr}\left(\boldsymbol{B}^{2}\right)\right]$. A função memória $M\left(t-t^{\prime}\right)$ é definida por

$$
M\left(t-t^{\prime}\right)=\sum_{k=1}^{m_{1}} \frac{a_{k}}{W_{i} \lambda_{k}} e^{-\left(\frac{t-t^{\prime}}{W_{i} \lambda_{k}}\right)},
$$

em que $W_{i}=\frac{\lambda_{r e f} U}{L}, \lambda_{k}$ são tempos de relaxação, $a_{k}$ são módulos de relaxamento e $m_{1}$ é o número de módulos de relaxação do polímero, determinadas à partir de dados experimentais. O número de modos depende do tipo específico de fluido mas, geralmente, 4 a 8 modos são suficientes para capturar dados viscoelásticos lineares, enquanto que, para soluções de polímeros 2 à 3 modos são suficientes (ver [11]). O histórico de deformação será calculado usando o método de campos de deformação proposto por [3]. Neste método, a deformação é convectada no tempo pela equação

$$
\frac{\partial}{\partial t} \mathbf{B}_{t_{i}^{\prime}}(\mathbf{x}, t)+\mathbf{u}(\mathbf{x}, t) \cdot \nabla \mathbf{B}_{t_{i}^{\prime}}(\mathbf{x}, t)=[\nabla \mathbf{u}(\mathbf{x}, t)]^{T} \cdot \mathbf{B}_{t_{i}^{\prime}}(\mathbf{x}, t)+\mathbf{B}_{t_{i}^{\prime}}(\mathbf{x}, t) \cdot \nabla \mathbf{u}(\mathbf{x}, t),
$$

considerando um numero finito $N$ de campos. Em cada iteração, os $N$ campos são redistribuídos no intervalo $I=[0, t]$ através de uma interpolação de segunda ordem. Para redistribuir os campos $t^{\prime}$ no intervalo $I$ será utilizada uma distribuição por progressão geométrica (ver [12])

\section{Método Numérico}

As equações (1)-(6) serão aproximadas em diferenças finitas em malhas cartesianas hierárquicas. Uma representação ilustrativa da malha é dada na Figura 1-(a) e sua estrutura de dependências é ilustrada na Figura 1-(b). Nesta estrutura de dados, cada célula pode ser particionada em formas geométricas distintas. Tal generalização impõe dificuldades na aproximação numérica em diferenças finitas. Por exemplo, considerando a Figura 1-(c), suponha que estamos interessados em aproximar a segunda derivada na direção $y$ centrada em $U_{c}$. Usando diferenças finitas de segunda ordem, temos:

$$
\frac{\partial^{2} U_{c}}{\partial y^{2}} \approx \frac{1}{\delta y}\left(U_{t}-2 U_{c}+U_{b}\right) ;
$$




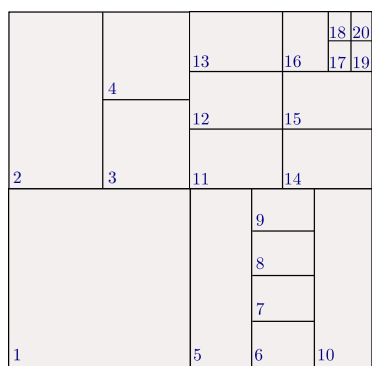

(a)

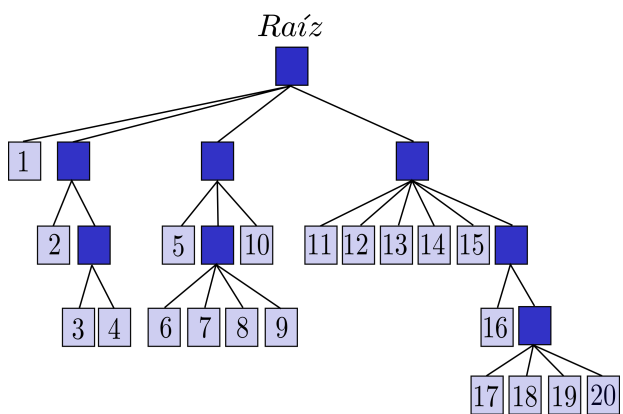

(b)

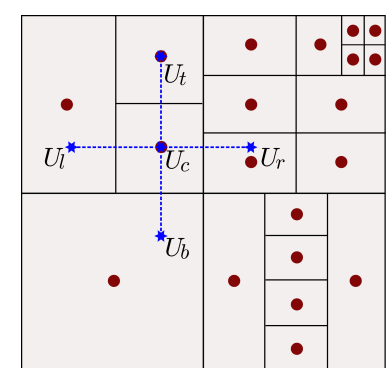

(c)

Figura 1: (a) Exemplo de malha cartesiana hierárquicas, (b) Estrutura hierárquicas, (c) Exemplo de aproximação em diferenças finitas.

Note que, neste caso, $U_{b}$ não coincide com valores conhecidos e será definida através da interpolação de valores das células vizinhas. Assim,

$$
U_{b}=\sum_{k=1}^{V_{b}} w_{k}^{b} U_{k} ;
$$

A quantidades de vizinhos $V_{b}$ é definida de acordo com a precisão imposta. Por exemplo, em uma aproximação de segunda ordem, como dada na equação $(7), V_{b}=6$ garante um erro de $3^{a}$ ordem na aproximação. Os pesos $w_{k}^{b}=w_{k}(\mathbf{x})$ são calculados utilizando mínimos quadrados móveis (MQM). Considerando um conjunto de $n$ funções interpoladoras suaves linearmente independentes $\boldsymbol{\Phi}_{i}=R^{d} \rightarrow R$, queremos interpolar o valor de $u$ tal que $U_{b}=U(\mathbf{x})=\sum_{k=1}^{n} c_{i} \boldsymbol{\Phi}_{i}(\mathbf{x})$.

Dado um conjunto $m$ de pontos conhecidos $\mathbf{x}_{1}, \mathbf{x}_{2}, \ldots, \mathbf{x}_{m} \in R^{d}$ com $m>n$ e $m$ valores $u_{1}=u\left(\mathbf{x}_{1}\right), u_{2}=u\left(\mathbf{x}_{2}\right), \ldots, u_{m}=u\left(\mathbf{x}_{m}\right)$, interpolar $u$ em $\mathbf{x}$ usando MQM consiste em minimizar o erro $E(\mathbf{c})$ onde

$$
E(\mathbf{c})=\|U-u\|_{2}^{2}=\sum_{i=1}^{m}\left(U\left(\mathbf{x}_{i}\right)-u_{i}\right)^{2} \frac{1}{\left\|\mathbf{x}-\mathbf{x}_{i}\right\|_{2}},
$$

ou, equivalentemente, $E(\mathbf{c})=\|W P \mathbf{c}-W \mathbf{u}\|_{2}^{2}$ onde $W=W(\mathbf{x})=\left\{\delta_{i j} \sqrt{\frac{1}{\left\|\mathbf{x}-\mathbf{x}_{i}\right\|_{2}}}\right\} \in R^{m \mathbf{x} m}$, $P=\left\{\boldsymbol{\Phi}_{j}\left(\mathbf{x}_{j}\right)\right\} \in R^{m \mathrm{x} n}$ e $\mathbf{u}=\left(u_{1}, u_{2}, \ldots, u_{m}\right)$.

A solução que minimiza o erro é dada por $\mathbf{c}(\mathbf{x})=(W P)^{\dagger} W \mathbf{u}$, onde $(\cdot)^{\dagger}$ denota a pseudo-inversa de Moore-Penrose (ver [9]).

Decompondo $(W P)$ em $Q R$ temos $\mathbf{c}(\mathbf{x})=R^{-1} Q^{t} W \mathbf{u}$. Assim,

$$
U(\mathbf{x})=\mathbf{c}^{t} \boldsymbol{\Phi}=\mathbf{u}^{t}\left(W Q R^{-t} \boldsymbol{\Phi}\right), \text { ou seja, } w_{k}=w_{k}(\mathbf{x})=W Q R^{-t} \mathbf{\Phi} .
$$

O procedimento para o cálculo de $w_{k}(\mathbf{x})$ deve ser realizado para cada aproximação $U(\mathbf{x})$, porém é feita uma única vez, visto que a malha é estática. 


\section{Resultados}

\subsection{Fluxo entre placas paralelas}

O escoamento entre placas paralelas foi simulado utilizando-se um fluido KBKZ 1módulo, com $\lambda_{1}=\lambda_{\text {ref }}=0.1396 \mathrm{~s}, a_{1}=1.6648 \mathrm{~Pa}, \rho_{0}=801.5 \mathrm{~kg} \mathrm{~m}^{-3}$ e $\eta_{0}=0.2324 \mathrm{~Pa} . \mathrm{s}$, $U=0.025 \mathrm{~ms}^{-1}$ e $L=1 \mathrm{~cm}$, o que fornece $R e=0.8621$ e $W i=0.3490$. Esse escoamento foi simulado nas malhas regulares $I$ de $80 \times 8$ (640 células ), $I I$ de $160 \times 16$ (2560 células), III de $320 \times 32$ (10240 células) e na malha $I V$ com refinamento local e um total de 3416 células. Para detalhes, ver figura 2.
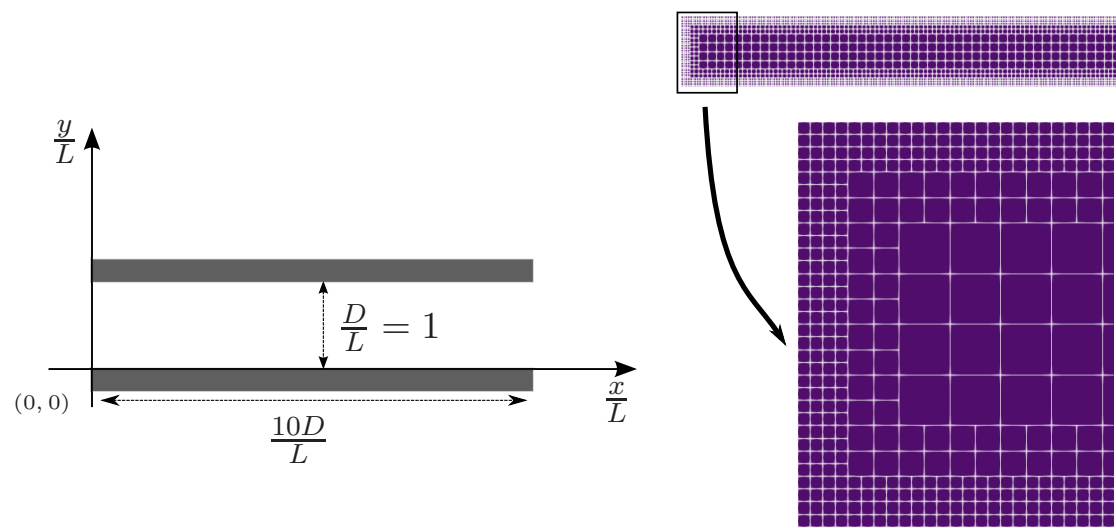

Figura 2: Domínio e representação da malha $I V$ com detalhe para a região de refinamento.

Os resultados obtidos e as respectivas soluções analíticas para a velocidade $u(y)$ e para os tensores $\tau^{x x}, \tau^{x y}$ e $\tau^{y y}$ são apresentados na Figura 3. Pode-se observar que existe boa concordância entre os resultados numéricos e as soluções analíticas que são apresentadas em [12]. Observa-se ainda que na malha $I V$ (com refinamento local) a solução numérica aproxima-se da solução da malha $I I I$ (malha mais fina), embora na região central as malhas $I$ e $I V$ apresentem as mesmas células.

Tabela 1: Erros na norma $L_{2}\left(* 10^{-2}\right)$

\begin{tabular}{ccccc}
\hline Malha & $u(y)$ & $\tau_{x x}$ & $\tau_{x y}$ & $\tau_{y y}$ \\
\hline$I$ & 0.271 & 0.025 & 0.108 & 0.154 \\
$I I$ & 0.057 & 0.007 & 0.071 & 0.043 \\
$I I I$ & 0.012 & 0.004 & 0.019 & 0.021 \\
$I V$ & 0.120 & 0.026 & 0.103 & 0.058 \\
\hline
\end{tabular}

Tabela 2: Erros na norma $L_{\infty}\left(* 10^{-2}\right)$

\begin{tabular}{ccccc}
\hline Malha & $u(y)$ & $\tau_{x x}$ & $\tau_{x y}$ & $\tau_{y y}$ \\
\hline$I$ & 0.310 & 0.046 & 0.111 & 0.200 \\
$I I$ & 0.063 & 0.013 & 0.062 & 0.047 \\
$I I I$ & 0.014 & 0.010 & 0.026 & 0.027 \\
$I V$ & 0.082 & 0.036 & 0.102 & 0.088 \\
\hline
\end{tabular}

Os erros cometidos na malhas $(I-I I I)$ e $I V$ são apresentados nas tabelas 1 e 2 . Nota-se que nas malhas $I-I I I$ o erro decresce com o refinamento em todas as variáveis (velocidade e tensores). O erro na malha $I V$ também é apresentado, embora não faça sentido a comparação direta entre o erro desta malha e o erro das outras malhas. Podese notar, porém, que o erro máximo para a velocidade $u(y)$ (ver tabela 2) diminuiu de 
$0.31010^{-2}$ (malha $I$ ) para $0.08210^{-2}$ (malha $I V$ ), o que já era indicado visualmente na região de ampliação na figura 3 ; ou seja, o refinamento próximo a parede melhorou a aproximação para a velocidade no centro do canal.

A velocidade converge com ordem aproximadamente 2 pois, considerando $L_{2}$, te$\operatorname{mos} \mathrm{O}(I I / I)=2.227$ e $\mathrm{O}(I I I / I I)=2.268$ e considerando $L_{\infty}$, temos $\mathrm{O}(I I / I)=2.282$ $\mathrm{e} \mathrm{O}(I I I / I I)=2.097$.


Figura 3: Escoamentos entre placas paralelas nas malhas I, II, III e IV.

\section{Conclusões}

Neste trabalho, apresentou-se uma nova metodologia usando Mínimos quadrados móveis para interpolar as variáveis (pressão, velocidade, tensor) em pontos onde os valores eram desconhecidos. A metodologia foi testada e verificada para o escoamento entre placas paralelas, considerando fluidos viscoelásticos governados pelo modelo integral KBKZ- 
PSM. A solução numérica foi comparada com uma solução semi-analítica e os resultados mostraram-se bons, tanto em malhas regulares quanto na malha com refinamento local.

\section{Agradecimentos}

Os autores agradecem à Fapesp (Processos 2017/21105-6 e 2013/07375-0) e à Petrobrás (Projeto 0050.0075367.12.9).

\section{Referências}

[1] M. Ansari, et al. Entry flow of polyethylene melts in tapered dies. International Polymer Processing, 25, (2010): 287-296.

[2] B. Bernstein, E. A. Kearsley, L. J. Zapas. A study of stress relaxation with finite strain, Journal of Rheology, 7, (1963), 391.

[3] M. A. Hulsen, E. Peters, B. V. D. Brule. A new approach to the deformation fields method for solving complex flows using integral constitutive equations, Journal of Non-Newtonian Fluid Mechanics, 98, (2001), 201-221.

[4] F. Losasso, F. Gibou, and R. Fedkiw. Simulating water and smoke with an octree data structure. ACM transactions on graphics (TOG). Vol. 23. No. 3. ACM, 2004.

[5] X-L. Luo and E. Mitsoulis. An efficient algorithm for strain history tracking in finite element computations of non-Newtonian fluids with integral constitutive equations. International Journal for Numerical Methods in Fluids, 11 (1990), 1015-1031.

[6] X-L. Luo and E. Mitsoulis. A numerical study of the effect of elongational viscosity on vortex growth in contraction flows of polyethylene melts. Journal of Rheology, 34, (1990): 309-342.

[7] E. Mitsoulis. 50 years of the K-BKZ constitutive relation for polymers. ISRN Polymer Science, 2013 (2013).

[8] A. C. Papanastasiou, L. E. Scriven, C. W. Macosko, An integral constitutive equation for mixed flows: viscoelastic characterization, Journal of Rheology, 27, (1983), 387.

[9] A. Quarteroni, R. Sacco, and F. Saleri, Numerical Mathematics. Springer, 2007.

[10] R. I. Tanner. From A to (BK)Z in constitutive relations. Journal of Rheology, v. 32, n. 7, p. 673-702, 1988.

[11] R. I. Tanner. Engineering rheology. Oxford University Press, 2000.

[12] M. F. Tomé, J. Bertoco, C. M. Oishi, M. S. B. Araujo, D. Cruz, F. T. Pinho e M. Vynnycky. A finite difference technique for solving a time strain separable k-bkz constitutive equation for two-dimensional moving free surface flows. J. Comput. Phys., 311, :114-141, 2016. DOI: 10.1016/j.jcp.2016.01.032. 